\title{
Guidelines for cochlear implantation in Saudi Arabia
}

Musaed A. Alzahrani, MBBS, MD, Nader F. Aldajani, MBBS, MD, Saeed A. Alghamdi, MBBS, MD.

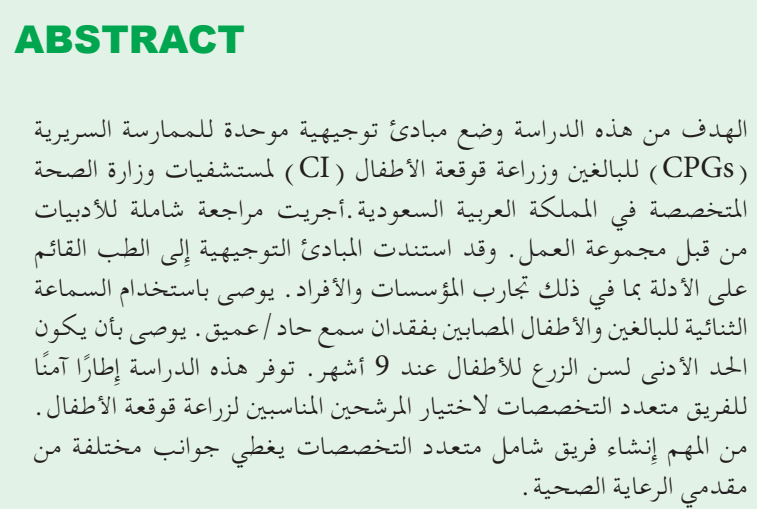

This study aim to establish unified clinical practice guidelines (CPGs) for adults and pediatric cochlear implantation (CI) for the Ministry of Health specialist hospitals in the Kingdom of Saudi Arabia. A comprehensive literature review was carried out by a task force group. Guidelines were based on evidencebased medicine including institutions and individuals' experiences. Bilateral CI is recommended for adults and pediatrics with bilateral severe/profound hearing loss. The minimum age of implantation for children is recommended at 9 months. This study provides a safe framework for the multidisciplinary team to select appropriate CI candidates. It is important to establish a comprehensive multidisciplinary team covering different aspects of health care providers.

Keywords: cochlear implant, guideline, hearing loss, single sided deafness, Kingdom of Saudi Arabia

Saudi Med J 2021; Vol. 42 (12): 1265-1271 doi: 10.15537/smj.2021.42.12.20210262

From the Department of Otolaryngology (Alzahrani), King Fahad Specialist Hospital, Dammam; from the Department of Otolaryngology (Aldajani), King Fahad Medical City, Riyadh; and from the Head and Neck, Skull Base Health Center (Alghamdi), King Abdullah Medical City, Makkah, Kingdom of Saudi Arabia

Received 5th July 2021. Accepted 10th October 2021.

Address correspondence and reprint request to: Dr. Musaed A. Alzahrani, Department of Otolaryngology, King Fahad Specialist Hospital, Dammam, Kingdom of Saudi Arabia. E-mail: dr.musaed@gmail.com ORCID ID https://orcid.org/0000-0002-7089-5677
A cochlear implant $(\mathrm{CI})$ is a device implanted surgically for advanced sensorineural hearing loss (SNHL). ${ }^{1}$ It has been recognized worldwide as the only acceptable cochlear rehabilitation technique for profound SNHL. ${ }^{2}$ The CI functions by transducing an acoustic signal into an electrical signal that directly stimulates the auditory nerve surviving spiral ganglia. In 1985, the Food and Drug Administration (FDA) approved the first multichannel CI for post-lingual SNHL in adults. Since then, advancements in technology, new surgical techniques, and modern programming strategies have expanded the candidacy criteria to include pre- and post-lingual SNHL in children. ${ }^{3}$ Worldwide, many center-based criteria and recommendations have been developed, ${ }^{4}$ which evolved into national clinical practice guidelines (CPGs) in different countries for funding CI programs. ${ }^{5}$ Clinical practice guidelines are statements that include recommendations intended to optimize patient care that are informed by a systematic review of evidence and an assessment of the benefits and harms of alternative care options. ${ }^{6}$

Since the beginning of CI in the Kingdom of Saudi Arabia (KSA) in the early 90s, the number of procedures has been growing exponentially and many centers around KSA have developed CI programs. Currently, there are 16 government hospitals with established CI centers. Ministry of Health $(\mathrm{MOH})$ hospitals are the major providers of CI with ten centers, followed by university hospitals (3 centers), and military hospitals (3 centers).

In 2020, the National Unified Procurement Company (NUPCO) became the main provider to most CI centers. National Unified Procurement Company has existed since 2009 and is owned by the Public Investment Bank, KSA. It specializes in purchasing, storing, and distributing medications, devices, and medical supplies through the unified procurement system for the benefit of government health sectors. This means that individual centers will no longer be able to purchase items directly through their hospitals' logistic departments. Although this may minimize the autonomy of each center, it is expected to result in a better outcome in terms of unified post-treatment 
follow-up care. This step has inspired us to work on developing CPGs to standardize the clinical services of $\mathrm{CI}$ in $\mathrm{MOH}$ specialist hospitals.

Rational for the CPGs. Throughout our research, we found no evidence of CPGs developed by any governmental entity before this study in KSA. This is most likely because the financial funding for CI centers is institution-dependent, and eventually, the multidisciplinary team of each center adopts a clinical practice based on its preference and best judgment. Therefore, representatives of the CI programs in King Fahad Medical City in Riyadh, King Abdullah Medical City in Makkah, and King Fahad Specialist Hospital in Dammam formed a task force to create unified CPGs for the participating centers.

Methods. A representative from the 3 participating centers was nominated for the task force to perform a comprehensive review of literature for CI program structure, settings, and required personnel. Candidacy criteria were also reviewed in different countries and recognized centers. The collected data were then referred to be assessed and evaluated by different team members including surgeons, audiologists as well as speech and language pathologists. Recommendations and guidelines were then recollected by the task force team. The final draft was resent and approved by each center.

Results. Establishing a CI program requires a multidisciplinary team approach with assigned roles in order to ensure the best outcomes. The number of implantations per year has to be proportionate to the available resources and personnel. Moreover, adequate and appropriate settings are necessary to run a successful program. This includes:

- Fully equipped, soundproof booths for behavioral assessment with air conduction (inserts, headphones, and speakers), bone conduction, and speech testing

- Objective testing equipment: immittance testing and otoacoustic emissions (OAEs)

-Electrophysiological testing equipment: auditory brainstem response (ABR) and auditory steady state response (ASSR)

- Basic and advanced vestibular testing equipment: videonystagmography (VNG), cervical vestibular evoked myogenic potentials (c-VEMP), Ocular -VEMP, and video head impulse test (vHIT)

- Necessary radiological exams: high-resolution CT and magnetic resonance imaging.
Members of the multidisciplinary CI program:

- Otologist: A specialized otolaryngologist head and neck surgeon who has undergone fellowship training in surgery of the temporal bone: the surgeon usually takes care of all medical preoperative assessments and referrals to other departments (such as: ophthalmology, cardiology, and genetics) as well as radiology and serology requests in order to investigate possible causes of hearing loss. They are also expected to counsel the parents/patients regarding the pre-operative and post-operative course.

-Audiologist: a well-trained audiologist with experience in behavioral and electrophysiological testing as well as programming and the verification of hearing aids and counselling during the preoperative course. In addition, an audiologist who has undergone specialized training in CI programming and troubleshooting is required for post-operative care. They play a major role in the CI candidacy process and long-term audiological and (re)habilitative follow-up.

- Speech and language pathologist (SLP): an SLP with specialized training in aural rehabilitation or auditory verbal therapy. The SLP plays a crucial role in assessing the candidates' auditory and communication skills and explaining the principles of and expectations from (re)habilitation, the importance of family involvement, and realistic expectations of post CI outcomes and progress. Patients should be offered a sufficient trial period to assess their potential benefit from appropriately fitted hearing aids.

- Psychologist and neuro-psychologist: they are essential for assessment and counseling of certain cases based on the patients' needs. For example, individuals on the autism spectrum or those with attention deficit hyperactivity disorders. Counseling parents or caregivers is also important because their involvement in the patient's rehabilitation and education is critical in order to achieve the best possible outcomes.

- Social worker: the social worker can provide guidance and support to the patient and the family in all areas, including financial planning when needed.

- Geneticist: the geneticist can be actively involved in the diagnosis process and family counseling.

- Other healthcare professionals: some patients require specialized services such as cardiology, ophthalmology, neurology, and developmental pediatrics. 
- Coordinator: the coordinator serves as the primary liaison representative between the patients or their families and the rest of the program team members. This includes resolving any enquiries they may have about the program as well as arranging any preoperative visits.

Cochlear implant pre-operative evaluation. Possible SNHL can be detected early in life through neonatal hearing screening programs using OAEs and automated ABR. ${ }^{7,8}$ Both screening tests are needed, especially for the detection of cases of auditory neuropathy spectrum disorder (ANSD). Neonates who fail screening are referred to specialized hospitals for diagnostic testing.

The pre-operative evaluation in children is carried out by the multidisciplinary team to determine the type and degree of hearing loss, if any, and assess the need for further management including the possibility of CI. In adults postlingual SNHL, the evaluation is less extensive and is limited most of the time to audiological, SLP and preoperative radiological assessment.

History and physical examination. A detailed and thorough history is mandatory when assessing a patient with hearing loss. This includes prenatal events (such, maternal infections and medications), mode of delivery, immediate post-natal health problems (such, hypoxia, jaundice, prolonged Neonatal Intensive Care Unit admission, and neonatal infections), and other medical conditions. Enquiring on family history of SNHL in parents, siblings, or relatives is also important. For adults, a complete history of the hearing loss should be obtained including the onset, progression, related symptoms (such as, tinnitus), and other otologic manifestations (such as, otorrhea and recurrent otitis media). Physical examination includes assessment of the external ear (such as, malformation, pits, or tags), otoscopic examination (such as, external auditory canal atresia or stenosis, cerumen impaction, and middle ear pathologies), examination for craniofacial dysmorphic features, oropharyngeal examination (such as, cleft lip or palate), and cervical examination (such as, branchial malformations and goiter).

Vestibular assessment. Sensorineural hearing loss could be associated with vestibular dysfunction due to the close proximity of the cochlea and the vestibule. Vestibular dysfunction is found in approximately $30-70 \%$ of children with hearing loss. ${ }^{9} 10$ For example, hearing and balance are often both affected in inner ear malformations (such, enlarged vestibular aqueduct, Mondini deformity, and common cavity) and in certain syndromes (such, Pendred syndrome and Usher syndrome). Recent theories also highlight the possibility of vestibular dysfunction after CI surgery. Using cVEMP to evaluate vestibular function post CI, Maes et $\mathrm{al}^{11}$ found a significant higher dysfunction in children with bilateral CI compared to children without a CI. Post CI vestibular dysfunction could be attributed to traumatic electrode insertion, foreign body autoimmune reaction, electrical stimulation, or labyrinthitis. ${ }^{12}$ In the majority of cases, post-operative dizziness subsides within a few weeks. ${ }^{13}$ For persistent dizziness, different tests are used to assess the vestibular function such as: VNG, caloric testing, cVEMP, oVEMP, vHIT, and the head shake test, with variable sensitivity and specificity. ${ }^{14}$

Radiological assessment. Temporal bone malformation is found in up to $30 \%$ of children with congenital SNHL. ${ }^{15}$ Magnetic resonance imaging is important to rule out auditory nerve (cranial nerve VIII) hypo/agenesis, inner ear dysplasia, or cochlear ossification. High-resolution CT (HRCT) provides better bony visualization of the inner ear and helps in identifying surgical landmarks, such as the large vestibular aqueduct, facial nerve course, position of the sigmoid sinus and dura, pneumatization of the mastoid bone and round window orientation. ${ }^{15}$ Although there is no global consensus of which radiologic modality is better for preoperative evaluation, we believe that both tests complement each other for better assessment.

Blood work. Serological examination includes the TORCH infections (toxoplasmosis, rubella, cytomegalovirus and herpes simplex virus) and syphilis. Thyroxin and thyroid-stimulating hormone levels may be tested as needed.

Ophthalmology consultation. There is a strong association between SNHL and ophthalmic pathologies in multiple syndromes. ${ }^{16,17}$ Moreover, assessing visual acuity is important as patients with SNHL are very dependent on this sense of communication. An experienced ophthalmologist may assess the visual acuity and perform an indirect fundoscopy for young children. Electroretinography (ERG) may be delayed till the age of 6 because retinal dystrophy is not usually detectable at young ages.

Electrocardiography. It is essential to rule out prolonged QT interval which assists in the differential diagnosis of Jervell and Lange-Nielsen Syndrome (JLNS). Although this is a rare syndrome, its high mortality rate warrants such non-invasive investigations that can be lifesaving.

Urinalysis and renal ultrasonography. Renal involvement is seen in some syndromic hearing loss cases, such as Alport syndrome and Branchiootorenal (BOR) syndrome. In cases of suspected BOR syndrome, 
renal ultrasound is performed routinely to exclude renal malformation.

Discussion. Most healthcare providers consider CI for patients with severe to profound SNHL not benefiting from conventional hearing aids. Global, national or regional guidelines exist in some countries such as the Western Australia clinical guidelines for adults and pediatrics CI and the British NICE guidelines. Other published CPGs includes the French SFORL, ${ }^{4}$ Belgium, ${ }^{18}$ Poland, ${ }^{19}$ and Spanish guidelines. ${ }^{20}$ However, the CPGs are modified regularly to include potential patients who are expected to do better with CI. After reviewing the above mentioned CPGs, a consensus agreement was achieved by the authors and their respective team members to establish the following CPGs.

Pediatric CI candidacy. The following criteria are our recommended CI indications for pediatrics: ${ }^{4,5,18-20}$ i) Bilateral severe to profound SNHL ( $>70 \mathrm{~dB}$ HL). ii) No benefit from the appropriately fitted hearing aid(s) for at least 3 to 6 months based on continued monitoring of the development of auditory skills, speech, and language. iii) No contraindication for surgery such as the absence of the cochlear nerve.

Sensorineural hearing loss is classified based on laterality (unilateral versus bilateral), the onset of hearing loss (pre-lingual, peri-lingual, and post-lingual), etiology (acquired or congenital), and severity (ranging from mild to profound). Wearing hearing aid(s), unless contraindicated, even in cases where no auditory benefit is anticipated has 2 essential advantages to the child; it provides the child with a sense of hearing and keeps the auditory nerve stimulated, thus enhancing the child's acceptance of wearing the CI's external device later on.

Bilateral CI has been proven to improve hearing with respect to noise and sound localization ability. ${ }^{21}$ It also provides superior results in speech perception and verbal intelligence. $^{22,23}$ In a systematic review of 21 studies, Lammer et $\mathrm{a}^{24}$ found that simultaneous CI yielded statistically significant improvement in outcomes for speech and language development compared to sequential CI. However, the outcomes among different inter-implant delay periods were inconclusive although longer periods seemed to have a negative effect on postoperative outcomes. Another review by Smulders et al ${ }^{25}$ concluded that implantation in both ears, simultaneous or sequential, before the age of 3.5 years is crucial for binaural pathway maturation of the auditory system. In children, an inter-implant delay of more than 24 months had a negative effect on speech performance scores. ${ }^{22,25}$ However, in adults, 4 out of 5 studies did not find a significant correlation between the speech scores and inter-implant delay. ${ }^{25}$ Moreover, the inter-implant delay did not show any correlation with localization after the second $\mathrm{CI}$ in all age groups. ${ }^{25}$

A comprehensive speech and language assessment is essential in the pre-candidacy process. It includes assessing the patient's current mode of communication (verbal or nonverbal,) as well as expressive and receptive speech and language skills. These are important when counseling regarding realistic expectations. In pre-lingual SNHL, a gap of more than 3 years between the child's chronological age and communication skills is expected to result in an overall poorer performance. Additionally, taking into consideration the family's education level and socioeconomic status, it is essential to ensure their commitment to the rehabilitation program.

According to the most recent FDA guidelines, the minimum age of implantation is 9 months in an otherwise healthy child. ${ }^{26}$ Placing an implant in a child as early as possible is recommended. ${ }^{27}$ Longitudinal outcomes of early implanted children resulted in improved speech and language skills and better functional performance, as well as positive effects on cognitive abilities and psychosocial development. ${ }^{28}$

Adults CI candidacy. The following criteria are our recommended CI indications for adults $>18$ years old: ${ }^{18-20}$ i) Bilateral simultaneous $\mathrm{CI}$ is the standard of care. ii) Post lingual bilateral severe SNHL (>70 dB HL) in the conversational frequencies $(1-4 \mathrm{kHz})$. Open-set sentence scores in less than $50 \%$ at an intensity of $65 \mathrm{~dB}$. iii) Limited or no benefit from the appropriately fitted hearing aid(s) as evident by aided thresholds or poor word recognition scores in the best-aided condition. iv) No contraindication for surgery such as injury of the vestibulocochlear nerve.

A recent review by Varadarajan et $\mathrm{al}^{29}$ found a more liberal recommendation for adults $\mathrm{CI}$ is being adopted in different publications. The adults CI criteria is expanding to include patients with greater levels of residual hearing and atypical causes of SNHL. Sladen et $\mathrm{al}^{30}$ found an improvement in hearing at quiet and noise for patients with short duration of unilateral SNHL less than 2 years. In another study, Marx et al, ${ }^{31}$ compared contralateral routing of signal (CROS) hearing aids, Bone anchored hearing aid (BAHA), and CI for patients with single sided deafness (SSD) and asymmetric SNHL. A significant improvement in quality of life especially in patients with associated severe tinnitus was noticed in CI recipients. Finally, off-criteria expansion was studied by Perkins et al, ${ }^{32}$ who included 105 patients with a higher consonant-nucleus-consonant (CNC) than conventional CI criteria and reported a significant 
benefit in noise and quiet. These data are examples of the need to increase general awareness about the potential benefits for CI to a more and wider range of SNHL subjects. Patients off-criteria who seek CI are entitled to be evaluated by the multidisciplinary CI team and benefit from CI accordingly.

Cochlear implant indications in asymmetric SNHL. It is defined as $>15 \mathrm{~dB}$ hearing loss (HL) interaural difference at 2 or more frequencies. ${ }^{33}$ In this group of patients, the objective is to restore bimodal auditory stimulation (hearing aid in the better ear and CI in the worse ear). ${ }^{20,34,35}$ Both adults and children can be candidates for this mode of stimulation. Candidacy for CI would be as follows: i) Severe to profound hearing loss in the conversational frequencies $(1-4 \mathrm{kHz})$ in the ear with the worse hearing capacity. In speech audiometry, open-set sentence scores in $<65 \%$. ii) Moderate to severe hearing loss in the conversational frequencies $(1-4 \mathrm{kHz})$ in the ear with the better hearing capacity. In speech audiometry, open-set sentence scores in $>85 \%$.

Electroacoustic CI candidacy. Electroacoustic stimulation, also known as hybrid implants, refers to the acoustic and electric stimulation of the same ear from one device. ${ }^{36}$ This mode of stimulation is indicated for adults with normal low-frequency hearing and profound high-frequency SNHL. ${ }^{37}$ Two companies provide electric-acoustic stimulation (EAS) systems; candidacy for each company would be as follows:

A) Cochlear hybrid system: ${ }^{36}$

i) Low frequency $(125-500 \mathrm{~Hz})$ hearing thresholds ranging from normal to $60 \mathrm{~dB} H \mathrm{HL}$ in the ear to be implanted.

ii) Mid to High-frequency hearing thresholds $>75 \mathrm{~dB}$ HL in the ear to be implanted.

iii) Word recognition score between $10 \%$ to $60 \%$ in the ear to be implanted and not more than $80 \%$ in the contralateral ear.

B) MED-EL EAS system: ${ }^{38}$

i) Normal to moderate SNHL up to the mid frequencies then sloping to severe/profound SNHL.

ii) Word score of $60 \%$ or less in the ear to be implanted.

\section{Special considerations}

- Cases of SNHL post-meningitis should be fitted with hearing aids as soon as possible and monitored closely. ${ }^{39}$ If hearing loss progresses to a severe/ profound level, bilateral implantation should be considered as soon as possible to avoid cochlear ossification. $^{40}$
- Temporal bone fractures may result in SNHL due to disruption of the sensory neuroepithelium leading to acute intracochlear hemorrhage and delayed labyrinthitis ossificans. $^{41}$ Early implantation is advised before the ossification appears. ${ }^{42}$

- Auditory neuropathy spectrum disorders (ANSD) require thorough investigations including $A B R$, OAEs, and electrocochleography. Hearing loss in ANSD may be due to pre- or post-synaptic alterations of inner hair cell function. Cases of pre-synaptic origin are reported to show better performance compared to those of post-synaptic origin..$^{43}$ Previous studies have shown variable long-term outcomes of CI in ANSD. ${ }^{43}$ In a recent study by Alzhrani et $\mathrm{al}^{44}$ no significant difference in auditory performance and speech intelligibility outcomes were noted between $\mathrm{CI}$ in otherwise healthy children and CI in children with isolated ANSD.

- Cochlear implant in SSD was initially introduced as a treatment for incapacitating tinnitus in SSD. ${ }^{45}$ Some authors recommend $\mathrm{CI}$ in patients whose tinnitus handicap inventory ${ }^{46}$ scores are more than 58 after the failure of other tinnitus therapeutic options. ${ }^{20}$ Later on, indications were expanded to include adults with acquired profound SSD. ${ }^{47}$ Sufficient data is not available on the benefits of CI in congenital SSD in children. ${ }^{47,48}$ Other rehabilitative options include CROS hearing aids and BAHA. ${ }^{49}$

- Cochlear implant in patients with autism spectrum disorder is controversial. ${ }^{50}$ Some reports demonstrated improved receptive function such as localization of sound and understanding simple commands. However, reported improvement in expressive functions was less prominent. Extensive family counseling with regards to realistic expectations is important. ${ }^{51,52}$

Post implant follow-up. Cochlear implant centers should provide post-implantation follow-up and rehabilitation to all CI recipients regardless of age, with different team members.

Surgical follow-up is recommended 10 days postoperatively for verification of wound healing and clearance for activation. This can be followed by annual or semiannual visits, as well as additional consultation as needed. Initial activation of the CI $\operatorname{system}(s)$ is recommended 2 to 4 weeks post-operatively. After initial activation, patients are typically seen at 1, 3, 6,9 , and 12 months post-activation in the first year, and at least every 6 months after that. Depending on the patient's age and auditory performance, follow-up visits may be scheduled on an annual basis. Audiology visits include assessment and programming which 
is very important to ensure optimal stimulation and performance. Aural (re)habilitation is often initiated 2 to 3 weeks post-activation, and is recommended at least once a week. Aural (re)habilitation sessions are tailored to each patient based on their performance and the family's active involvement. These sessions are delivered for approximately 3 to 4 years or till the patient is of school-age if the school provides proper SLP follow-up.

In conclusion, CPGs provide a safe framework for the multidisciplinary team to select appropriate CI candidates. Bilateral simultaneous $\mathrm{CI}$ is the standard of care and implantation is advised as young as possible. Atraumatic approach is advised in order to spare the residual cochlear and vestibular function.

Acknowledgment. The authors acknowledge the support of the Saudi Society of Otolaryngology-Head and Neck Surgery. Also, we acknowledge and appreciate Dr. Tarek Eldesouki, Dr. Saeed Thabet, Dr. Nad E. Kattan, Dr. Demah Almowanes, and Ms. Omnia Arab for their valuable input to this paper. We would like to thank as well the True Editors Limited (www.trueeditors.com) for English language editing.

\section{References}

1. Deep NL, Dowling EM, Jethanamest D, Carlson ML. Cochlear implantation: an overview. J Neurol Surg B Skull Base 2019; 80: 169-177.

2. Foteff C, Kennedy S, Milton AH, Deger M, Payk F, Sanderson G. Cost-utility analysis of cochlear implantation in Australian adults. Otol Neurotol 2016; 37: 454-461.

3. Carlson ML, Sladen DP, Gurgel RK, Tombers NM, Lohse CM, Driscoll CL. Survey of the American Neurotology Society on cochlear implantation: Part 1, candidacy assessment and expanding indications. Otol Neurotol 2018; 39: e12-e19.

4. Simon F, Roman S, Truy E, Barone P, Belmin J, Blanchet $\mathrm{C}$, et al. Guidelines (short version) of the French society of otorhinolaryngology (SFORL) on pediatric cochlear implant indications. Eur Ann Otorhinolaryngol Head Neck Dis 2019; 136: 385-391.

5. Vickers D, De Raeve L, Graham J. International survey of cochlear implant candidacy. Cochlear Implants Int 2016; 17 : 36-41.

6. American Academy of Family Physicians. Clinical Practice Guideline Manual. [Updated 2021; accessed 2021 July 19]. Available from: https://www.aafp.org/family-physician/patientcare/clinical-recommendations/cpg-manual.html

7. Kanji A, Khoza-Shangase K, Moroe N. Newborn hearing screening protocols and their outcomes: A systematic review. Int J Pediatr Otorhinolaryngol 2018; 115: 104-109.

8. Wroblewska-Seniuk KE, Dabrowski P, Szyfter W, Mazela J. Universal newborn hearing screening: methods and results, obstacles, and benefits. Pediatr Res 2017; 81: 415-422.

9. Shinjo Y, Jin Y, Kaga K. Assessment of vestibular function of infants and children with congenital and acquired deafness using the ice-water caloric test, rotational chair test and vestibularevoked myogenic potential recording. Acta Otolaryngol (Stockh) 2007; 127: 736-747.
10. Fernandes R, Hariprasad S, Kumar VK. Physical therapy management for balance deficits in children with hearing impairments: A systematic review. I Paediatr Child Health 2015; 51: 753-758.

11. Maes L, De Kegel A, Van Waelvelde H, Dhooge I. Rotatory and collic vestibular evoked myogenic potential testing in normalhearing and hearing-impaired children. Ear Hear 2014; 35: e21-32.

12. Murray D, Viani L, Garvan J, Murphy A, Vance R, SimoesFranklin C, et al. Balance, gait and dizziness in adult cochlear implant users: A cross sectional study. Cochlear Implants Int 2020; 21: 46-52.

13. Kubo T, Yamamoto K, Iwaki T, Doi K, Tamura M. Different forms of dizziness occurring after cochlear implant. Eur Arch Otorhinolaryngol 2001; 258: 9-12.

14. Verbecque E, Marijnissen T, De Belder N, Van Rompaey V, Boudewyns A, Van de Heyning P, et al. Vestibular (dys)function in children with sensorineural hearing loss: a systematic review. Int J Audiol 2017; 56: 361-81.

15. Yigit O, Kalaycik EC, Yasak AG, Araz SE. Which imaging modality in cochlear implant candidates? Eur Arch Otorhinolaryngol 2019; 276: 1307-1311.

16. Abou-Elhamd KA, ElToukhy HM, Al-Wadaani FA. Syndromes of hearing loss associated with visual loss. Eur Arch Otorhinolaryngol 2014; 271: 635-646.

17. Alzahrani M, Tabet P, Saliba I. Pediatric hearing loss: common causes, diagnosis and therapeutic approach. Minerva Pediatr 2015; 67: 75-90.

18. De Raeve L. Cochlear implants in Belgium: Prevalence in paediatric and adult cochlear implantation. Eur Ann Otorhinolaryngol Head Neck Dis 2016; 133: S57-S60.

19. Szyfter W, Karlik M, Sekula A, Harris S, Gawęcki W. Current indications for cochlear implantation in adults and children. Otolaryngol Pol 2019; 73: 1-5.

20. Manrique M, Ramos Á, de Paula Vernetta C, Gil-Carcedo E, Lassaletta L, Sanchez-Cuadrado I, et al. Guideline on cochlear implants. Acta Otorrinolaringol Esp 2019; 7: 47-54.

21. Jang JH, Roh J-M, Choo OS, Kim Y-J, Kim H, Park HY, et al. Critical factors for binaural hearing in children with bilateral sequential cochlear implantation: first implant performance and inter-implant interval. Audiol Neurootol 2019; 24: 174-82.

22. Gordon KA, Papsin BC. Benefits of short interimplant delays in children receiving bilateral cochlear implants. Otol Neurotol 2009; 30: 319-331.

23. Gordon KA, Jiwani S, Papsin BC. What is the optimal timing for bilateral cochlear implantation in children? Cochlear Implants Int 2011;12: S8-S14.

24. Lammers MJW, Venekamp RP, Grolman W, van der Heijden GJMG. Bilateral cochlear implantation in children and the impact of the inter-implant interval. Laryngoscope 2014; 124: 993-999.

25. Smulders YE, Hendriks T, Stegeman I, Eikelboom RH, Sucher C, Upson G, et al. Predicting sequential bilateral cochlear implantation performance in postlingually deafened adults; A retrospective cohort study. J Otol Rhinol 2018; 43: 1500-1507.

26. Friedmann DR, Tona KM, Roland JT, Spitzer ER, Waltzman SB. Cochlear implantation in children under 12 months: Prevalence and implications of "hidden" disabilities. Cochlear Implants Int 2020; 21: 307-312.

27. Leigh JR, Dettman SJ, Dowell RC. Evidence-based guidelines for recommending cochlear implantation for young children: Audiological criteria and optimizing age at implantation. Int J Audiol 2016; 55: S9-S18. 
28. Ching TYC, Dillon H, Leigh G, Cupples L. Learning from the longitudinal outcomes of children with hearing impairment (LOCHI) study: summary of 5-year findings and implications. Int J Audiol 2018; 57: S105-S111.

29. Varadarajan VV, Sydlowski SA, Li MM, Anne S, Adunka OF. Evolving criteria for adult and pediatric cochlear implantation. Ear Nose Throat J 2021; 100: 31-37.

30. Sladen DP, Carlson ML, Dowling BP, Olund AP, Teece K, DeJong $\mathrm{MD}$, et al. Early outcomes after cochlear implantation for adults and children with unilateral hearing loss. Laryngoscope 2017; 127: 1683-1688.

31. Marx M, Mosnier I, Venail F, Mondain M, Uziel A, Bakhos $\mathrm{D}$, et al. Cochlear implantation and other treatments in singlesided deafness and asymmetric hearing loss: results of a national multicenter study including a randomized controlled trial. Audiol Neurootol 2021; 1-11.

32. Perkins E, Dietrich MS, Manzoor N, O’Malley M, Bennett M, Rivas A, et al. Further evidence for the expansion of adult cochlear implant candidacy criteria. Otol Neurotol 2021; 42: 815-823.

33. Durakovic N, Valente M, Goebel JA, Wick CC. What defines asymmetric sensorineural hearing loss? Laryngoscope 2019; 129: 1023-1024.

34. Wilson K, Ambler M, Hanvey K, Jenkins M, Jiang D, Maggs J, et al. Cochlear implant assessment and candidacy for children with partial hearing. Cochlear Implants Int 2016; 17: 66-99.

35. Dillon MT, Buss E, Rooth MA, King ER, McCarthy SA, Bucker AL, et al. Cochlear implantation in cases of asymmetric hearing loss: subjective benefit, word recognition, and spatial hearing. Trends Hear 2020; 24: 2331216520945524.

36. Woodson EA, Reiss LAJ, Turner CW, Gfeller K, Gantz BJ. The hybrid cochlear implant: A review. Adv Otorhinolaryngol 2010; 67: 125-134.

37. Li C, Kuhlmey M, Kim AH. Electroacoustic stimulation. Otolaryngol Clin North Am 2019; 52: 311-322.

38. Pillsbury HC, Dillon MT, Buchman CA, Staecker H, Prentiss SM, Ruckenstein MJ, et al. Multicenter US clinical trial with an Electric-Acoustic Stimulation (EAS) system in adults: final outcomes. Otol Neurotol 2018; 39: 299-305.

39. Karanja BW, Oburra HO, Masinde P, Wamalwa D. Prevalence of hearing loss in children following bacterial meningitis in a tertiary referral hospital. BMC Res Notes 2014; 7: 138.

40. Rodenburg-Vlot MBA, Ruytjens L, Oostenbrink R, van der Schroeff MP. Repeated audiometry after bacterial meningitis: consequences for future management. Otol Neurotol 2018; 39: e301-e306.
41. Trakimas DR, Knoll RM, Ishai R, Lee DJ, Jung DH, Nadol $\mathrm{JB}$, et al. Otopathology of unilateral cochlear implantation in patients with bilateral temporal bone fracture. Otol Neurotol 2019; 40: e14-e19.

42. Vermeire K, Brokx JPL, Dhooge I, Heyning PHV de. Cochlear implantation in posttraumatic bilateral temporal bone fracture. ORL J Otorhinolaryngol Relat Spec 2012; 74: 52-56.

43. Anderson P, Schaefer S, Henderson L, Bruce IA. Cochlear implantation in children with auditory neuropathy: Lessons from Brown-Vialetto-Van Laere syndrome. Cochlear Implants Int 2019; 20: 31-38.

44. Alzahrani M, Tabet P, Saliba I. Pediatric hearing loss: common causes, diagnosis and therapeutic approach. Minerva Pediatr 2015; 67: 75-90

45. Van de Heyning P, Vermeire K, Diebl M, Nopp P, Anderson I, De Ridder D. Incapacitating unilateral tinnitus in single-sided deafness treated by cochlear implantation. Ann Otol Rhinol Laryngol 2008; 117: 645-652.

46. Newman CW, Jacobson GP, Spitzer JB. Development of the tinnitus handicap inventory. Arch Otolaryngol Head Neck Surg 1996; 122: 143-148.

47. Arndt S, Laszig R, Aschendorff A, Hassepass F, Beck R, Wesarg T. Cochlear implant treatment of patients with single-sided deafness or asymmetric hearing loss. HNO 2017; 65: 98-108.

48. Thomas JP, Neumann K, Dazert S, Voelter C. Cochlear implantation in children with congenital single-sided deafness. Soc Eur Acad Otol Neurotol 2017; 38: 496-503.

49. Kim G, Ju HM, Lee SH, Kim H-S, Kwon JA, Seo YJ. Efficacy of bone-anchored hearing aids in single-sided deafness: A systematic review. Otol Neurotol 2017; 38: 473-483.

50. Lachowska M, Pastuszka A, Łukaszewicz-Moszyńska Z, Mikołajewska L, Niemczyk K. Cochlear implantation in autistic children with profound sensorineural hearing loss. Braz J Otorhinolaryngol 2018; 84: 15-19.

51. Eshraghi AA, Nazarian R, Telischi FF, Martinez D, Hodges A, Velandia $S$, et al. Cochlear implantation in children with autism spectrum disorder. Otol Neurotol 2015; 36: e121-e128.

52. Donaldson AI, Heavner KS, Zwolan TA. Measuring progress in children with autism spectrum disorder who have cochlear implants. Otol Neurotol 2004; 130: 666-671. 